Historic, Archive Document

Do not assume content reflects current scientific knowledge, policies, or practices. 



\section{PEONY PRICE LIST}

\section{for 1927-8}

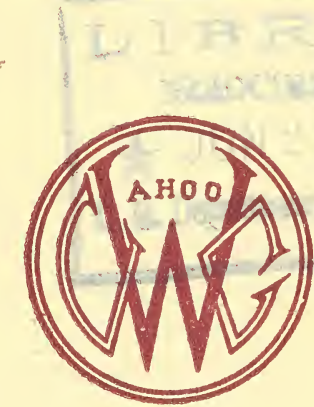

\section{Wahoo Lodge Gardens}

से

Sioux Falls, - South Dakota P. O. Box 915 


\section{TERMS}

Our terms are cash with order, unless other arrangements have been made. We never substitute unless requested to do so.

Should we be sold out of the variety ordered we will promptly return your money.

\section{GUARANTEE}

All plants sent out by us are guaranteed true to name and in good healthy condition. Should they prove otherwise, same will be replaced free of charge and customer may keep the roots sent in the first place.

\section{PRICES}

Prices quoted are for strong natural divisions of Two, Three or More Blooming size eyes, the accepted size best suited for all purposes.

Should any certain size be desired other than specified we can supply them, and oftentimes at prices more attractive than listed elsewhere.

\section{SHIPMENT}

Shipments will be made by Express collect unless otherwise instructed. If by parcel post add for each root 10 cents to cover insured delivery.

\section{COLLECTIONS AND DISCOUNTS}

We offer no Collections, but shall be pleased to offer suggestions and special prices to those purchasing for the first time as to the varieties best suited for the beginner, but those who are familiar with and adding to their list of Peonies, we feel should make their own selection in which case we will allow you to select $\$ 11.00$ worth of roots for $\$ 10.00$, $\$ 27.50$ worth for $\$ 25.00$, $\$ 57.50$ worth for $\$ 50.00, \$ 120.00$ worth for $\$ 100.00$.

\section{SOIL AND CLIMATE}

Our Soil and Climatic Conditions in this country are the best in the world for propagating Peonies, therefore our Peony roots cannot be excelled.

\section{LOCATION}

Our Exhibition Gardens are located two miles south of the city of Sioux Falls on State and Federal highway No. 15.

No description equals seeing the different varieties in bloom. Thousands avail themselves of the opportunity every season. Visitors always welcome. 
8.5 Adolph Rosseau, Dark Red.....\$1.50 \$3.50

8.6 Albert Crousse, Sal. Pink....... 1.00

2.50

7.9 Alfred De Musset, Blush White.. 1.00

2.50

7.2 Alice De Juvlecourt, White..... 1.00

1.25

8.8 Alsace-Lorraine, Cream White.. 3.00

7.50

7.6 Archie Brand, Shell Pink....... 1.50

3.25

7.7 Adelaide E. Hollis, Rose White... 2.75

6.50

8.8 Albatre (Avalanche), Ivory White 1.00

2.50

7.1 Alexander Dumas, Violet Rose... 1.00

1.50

7.1 Alexandriana, Light Rose....... 1.00

2.00

8.2 Amanda Yale, White.......... 1.00

2.50

7.2 American Beauty, Fine Red..... 1.00

2.00

7.7 Armandine Mechin, Dark Crimson 1.00

2.00

8.6 Asa Gray, Pale Lilac........... 1.00

2.50

7.5 Atrosanguinea, Dark Red....... 1.00

2.25

8.7 Auguste Dessert, Rich Rose Red..10.00 25.00

7.3 Auguste Villaume, Violet Rose... 1.00

7.8 Augustin D'Hour, Bril. Red..... 1.00

8.1 Aurore, Lilac White.......... 1.00

2.50

2.50

8.0 Aviateur Reymond, Cherry Red.. 2.00

9.1 Baroness Schroeder, Fl. White... 1.50

8.5 Bayadere, Creamy White...... 4.00

7.2 Beaute D'Villicants ........... 1.00

7.9 Beauty's Mask, Blush-White.... 1.50

8.6 Bertrade, White ............ 3.00

7.0 Berlioz, Carmine Rose......... 1.00

8.0 Boule De Neige, White........ 1.00

Beatrice Kelway, Rose and Yellow 1.00

9.00

2.50

3.50

7.25

2.50

2.50

2.00

8.1 Benj. Franklin, Maroon......... 1.00

2.50

8.2 Brand's Magnificent, Red....... 3.75

9.00

7.5 Bunch of Perfume, Rose....... 1.00

7.2 De Condolle, Purple.......... 1.00

2.00

7.6 Bunker Hill, Rose (Tyrian) ..... 2.50

7.1 Candidissima, White ........ 1.00

2.50

6.00

8.4 Candeur, Silvery Rose......... 5.00

7.8 Carnea Elegans, Flesh Pink..... 1.50

7.8 Chas. McKellep, Ruby Crimson.. 2.00

7.9 Chas. S. Minot, Shell Pink..... 2.00

2.00

12.00

2.50

4.75

5.00

7.6 Charlotte Cushman, Red........ 2.00

8.6 Cherry Hill, Garnet........ 5.00

8.4 Chestine Gowd, Shell Pink..... 2.00

8.7 Claire Dubois, Rose Pink....... 1.00

5.00

12.00

4.75

8.5 Clemenceau, Carmine ........6. 6.00

2.50

7.5 Constant Devred, Rose Carmin.. 1.00

14.00

7.2 Coquelin, Tyrian Rose....... 1.00

2.50

2.50 
8.5 Coronation, Pale Pink......... 3.00

7.25

8.1 Couronne D'or, White........ 1.00

2.50

8.4 David Harem, Bright Red...... 7.00

16.50

7.0 Deemster, Choc. Maroon........ 1.50

3.50

7.1 Delachei, Crimson ........... 1.00

2.50

7.6 Delicatissima, Rose .......... 1.00

2.00

7.7 Dorchester Pink ............ 1.00

1.50

8.1 Dr. H. Barnsby, Red........ 2.00

4.75

7.8 Duc De Wellington, White..... 1.00

8.3 Duchesse De Nemours, White... 1.00 2.00

7.3 Duke of Devonshire, Crimson.... 1.00

7.7 Edouard Andre, Violet Red..... 1.00

2.25

2.50

7.9 Edulis Superba, Mauve Pink.... 1.00

7.5 Edwin Forest, Dark Crimson.... 2.00

9.5 Eliz. B. Browning, Pink White.. 5.00

8.1 E. G. Hill, Tyrian Rose....... 2.50

7.3 Ella C. Kelway, Lav. Flesh.... 1.00

9.0 Elwood Pleas, Light Rose...... 3.00

2.00

4.75

12.00

6.00

2.00

7.25

7.6 Ella Wheeler Wilcox, Pink..... 1.00

2.25

Emma, Blush Rose.......... 3.50

8.50

8.0 Enchantment, Lilac Rose........ 1.50

3.50

8.6 Enchantress, Creamy White..... 5.00

11.50

8.5 Estafette, Purple, Carm........ 2.00

7.5 Etta, Shell Pink............. 1.50

4.75

3.50

7.6 Eucharis, Cream White....... 2.50

7.9 Eugene Bigot, Tyrian Rose..... 1.50

6.00

3.50

8.3 Eugene Verdier, Pale Pink..... 2.00

4.75

8.5 Eugeine Verdier, Pink......... 1.00

2.25

4.75

7.3 Eugene Reignoux, Pink....... 2.00

8.2 Evangeline, Pale Rose......... 3.50

8.3 Evening Glow, Blush Pink..... 1.50

8.50

8.5 Exquisite Kelway's, Deep Pink... 3.00

7.7 Fanny Crosby, Soft Pink...... 2.50

8.2 Fairbault, Deep Rose......... 2.00

8.4 Felix Crousse, Brilliant Red.... 1.00

9.3 Festiva Maxima, Pure White... 1.00

7.5 Floral Treasure, Pale Rose..... 1.00

8.1 Florence Nightingale, White.... 1.50

8.3 Fraicheur, Pink and Cream..... 2.50

9.1 Francis Willard, White....... 2.25

8.7 Frances Shaylor, White and Yellow .................... 5.00

3.50

7.25

6.00

4.75

2.50

2.50

2.25

3.75

6.00

4.50

12.00

8.2 Francois Rousseau, Red........ 2.50

6.00

8.1 Galathea, Flesh White........ 3.50

7.5 Gen. Bertrand, Red.......... 1.00 
7.7 George Hollis, Pink and White.. 2.00

7.2 George Washington, Crimson... 1.00

2.50

George W. Tyron, Rose....... 2.00

3.75

8.9 Georgiana Shaylor, Pink....... 3.50

6.00

Geraldine, Splendid Pink \& Yellow 1.00

2.50

8.5 Germaine Bigot, Rose......... 1.50

3.50

8.0 Gigantica, Large Bright Pink.... 1.00

2.50

8.8 Ginette, Salmon and Blush Pink.. 3.00

7.25

8.8 Gisele, Flesh White......... 2.50

8.1 Gismonda, Pink ............ 1.50

7.9 Glory of Summerset, Pink...... 1.00

7.9 Gloire De Chas. Gombault, Light

Rose ....................1.00

6.00

3.50

2.50

8.1 Grandiflora Nivea Plena, Milk

White .................... 1.00

7.3 Golden Harvest, Pink.......... 1.00

8.8 Grandiflora, Rose White....... 1.50

8.2 Grover Cleveland, Dark Crimson. 1.50

8.2 H. A. Hagen, Deep Rose...... 3.50

7.9 Harriet Farnsley, Soft Pink..... 1.00

7.7 H. F. Reddick, Crimson........ 1.25

7.9 Helen Wolaver, Shell Pink..... 2.00

8.8 Henry Avery, White.......... 6.00

9.2 Jeannot, Flesh Pink...........10.00

7.9 Henri Demay, Aniline Red...... 1.00

8.7 James Kelway, Rose White..... 1.50

8.3 Jeanne Gaudichau, White...... 2.00

8.6 Jessie Shaylor, Deep Red...... 8.00

7.9 Jeanne D'Arc, Pink and White.. 1.00

8.1 John Richardson, Large Deep Pink 2.00

9.2 Jubilee, Grand White......... 3.00

8.6 Judge Berry, Flesh Pink....... 5.00

8.9 Karl Rosenfield, Crimson....... 1.50

9.4 Kelway's Glorious, Magnificent

White .................... 15.00

8.5 Kelway's Queen Pink...........12.50

Kelway's False Queen......... 1.00

9.3 Lady Alexander Duff, Pale Rose.. 2.75

8.2 Lady Beresford, Blush Pink..... 1.50

7.0 Lady Curzon, Lilac White...... 1.00

Lady Mayoress, Rose and White. 1.00

2.50

2.50

2.50

2.50

2.50

8.50

2.50

3.00

4.75

12.50

24.00

2.50

3.50

4.75

19.00

2.50

4.75

6.25

12.00

3.50

36.00

30.00

2.50

5.75

3.50

2.00

2.00

$7.8 \mathrm{La}$ Fayette, Rich Pink........ 1.75

9.2 La Fee, Rose Pink............. 10.00

4.25

24.00

8.6 La Fiancee, Creamy White..... 4.00

9.0 La France, Rose White....... 5.00

9.50

12.00 
9.1 La Lorraine, Creamy White..... $5.00 \quad 12.00$

7.3 L'Indispensable, Light Pink..... $1.00 \quad 2.00$

7.9 La Martine (Gigantea), Pink ... $1.00 \quad 2.50$

8.0 La Martine (Lemoine), Carmine. $5.00 \quad 12.00$

8.5 La Perle, Lilac White......... $1.00 \quad 2.50$

8.3 La Rosiere, Cream White....... $1.00 \quad 2.50$

8.1 La Tendresse, White and Crimson $1.00 \quad 2.50$

$7.5 \mathrm{La}$ Tulipe, Cream White...... $1.00 \quad 2.00$

8.8 Laura Dessert, White.......... $7.00 \quad 17.00$

9.9 Le Cygne, Milk White.........10.00 24.00

8.4 Livingstone, Rose Pink......... $1.00 \quad 2.50$

7.5 Little Sweetheart, Salmon Pink. 1.002 .25

7.8 Louisa Brand, Blush White..... $3.50 \quad 8.50$

9.0 Longfellow, Cherry Crimson..... 4.0010 .50

8.4 Laura Dexheimer, Flaming Crimson ....................... 2.00

7.8 Lord Kitchener, Cherry Red.... 2.00 4.75

8.8 Loveliness, Hydrangea Pink..... 3.00

8.7 Luetta Pfeiffer, Pinkish White... 7.25

8.1 Lucy E. Hollis, Lilac Rose...... 1.50

8.6 Mme. Auguste Dessert, Rose.... 1.50

7.4 Mme. Breon, Pink .......... 1.00

8.0 Mme. Benoit Riviere, Soft Rose. 3.00

7.8 Mme. Barillet Deschamps, Lilac

Pink ................... 1.00

7.3 Mme. Bucquet, Crimson ....... 1.00

8.1 Mme. Calot, Hydrangea Pink.... 1.00

7.3 Mme. Camille Bancel, Deep Pink 1.00

7.9 Mme. Crousse, Pure White..... 1.00

7.4 Mme. De Galhau, Rose White... 1.00

8.2 Mme. De Treyeran, Rose White. 2.00

7.7 Mme. De Vatry, Lilac White... 1.00

7.9 Mme. De Verneville, Pink White. 1.00

8.0 Mme. Ducel, Soft Pink........ 1.00

8.5 Mme. Emile Galle, Lilac White.. 1.00

8.9 Mme. Emil Lemoine, Flesh White 1.50

8.4 Mme. Emile Dupraz, Carmine Pink 4.00

7.7 Mme. Forel, Dark Pink........ 1.00

7.9 Mrs. Jeanne R. Gowdy, Ruffled

Pink ...................... 3.00

8.9 Mme. Emile Lemoine, Milk White 1.50

7.8 Mme. Lemoine, Pink ......... 1.50

8.0 Mme. Lemoinier, Lilac White.... 1.00

7.4 Mme. Fould, Late White........ 1.00

7.7 Mme. Guyot, Pink and Yellow.. 2.50

4.75

7.00

17.50

3.50

3.75

1.50

7.25

2.50

2.50

2.50

2.50

2.50

2.00

4.75

2.50

2.50

2.50

2.50

3.50

9.50

2.50

7.25

3.50

3.50

2.50

2.50

6.00 
7.9 Mme. Geissler, Violet Rose..... 1.00

7.7 Mme. Herve, Solferino-Red .... 1.00

8.8 Mme. Gaudichau, Intense Crimson 5.75

7.9 Mme. Joanne Sallier, Violet Rose. 3.50

7.5 Mme. Moutot, Tyrian Rose..... 1.00

9.4 Mme. Jules Dessert, Blush and

Salmon ................... 3.00

2.50

8.5 Mme. Manchet, Silvery Lace Lilac 1.50

7.3 Mme. Reignoux, Rose-Carmine .. 1.50

7.7 Mme. Savreau, Pink and Cream. 2.00

8.2 Mme. Jeanne Riviere, Flesh Pink. 2.50

8.0 Mlle. Leone Calot (M. Chas.

Leveque), Salmon Pink........ 1.00

7.3 Mlle. Marie Calot, Flesh........ 1.00

8.4 Mlle. Rousseau, Blush White.... 1.50

8.1 Marcelle Dessert, White and Lilac 1.50

8.6 Marchioness of Landowne, $\mathrm{Hy}$ -

drangea Pink .............. 2.50

8.2 Marguerite Gaudichau, Shell Pink 3.00

8.6 Marguerite Gerard, Flesh Pink.. 1.00

9.0 Marie Crousse, Delicate Pink.... 2.00

7.9 Marie D'Hour, China Pink..... 1.00

8.3 Marie Jacquin, Delicate Flesh

Color .....................25

8.5 Marie Lemoine, Large White... 1.00

7.8 Marquis C. Lagergren, Cherry Red 1.50

7.5 Marshall Vaillant, Deep Rose... 1.00

9.1 Martha Bullock, Rose Pink......10.00

8.7 Mary Brand, Dark Red....... 2.00

8.0 Mary L. Hollis, Lilac Rose..... 2.00

9.0 Mary Woodbury Shaylor, Clear

Pink .................... 10.00

7.4 Masterpiece, Bright Rose...... 1.00

7.9 Mathilde De Roseneck, Pale Rose 1.00

8.5 Maud L. Richardson, Rose...... 2.50

7.2 Meissonier, American Beauty.... 1.00

7.9 Midnight, Maroon .......... 1.00

7.25

3.50

3.50

4.75

6.00

2.50

2.50

3.50

3.50

6.00

7.25

2.50

4.75

2.50

3.00

2.50

3.50

2.25

24.00

4.75

4.75

24.00

2.25

2.25

2.25

2.50

8.3 Midsummer Night's Dream, Ivory

White ..................... 2.50

9.0 Milton Hill, Lilac Rose........ 4.75

Mignon, Amber Cream........ 3.75

7.8 Miss Salway, Lilac White...... 4.75

7.8 Modeste Guerin, Deep Pink.... 1.00

7.8 Modele De Perfection, Violet Rose 1.00

8.3 Mons. Dupont, Milk White..... 1.00

6.00

11.50

9.00

11.50

2.25

2.25

2.25 
7.7 Mons. Krelage, Dark Red...... $1.00 \quad 2.50$

9.2 Mons. Jules Elie, Shell Pink..... $1.50 \quad 3.50$

9.0 Mons. Martin Cahuzac, Dark

Garnet ................... $2.50 \quad 6.00$

8.4 Mont Blanc, White Tinted Rose. 2.50 6.00

7.8 Mrs. Carew, Silvery Blush...... $1.50 \quad 3.50$

8.3 Mrs. A. G. Ruggles, White and

Lilac ...................... 2.75

9.3 Mrs. Ed. Harding, White......15.00

8.3 Mrs. Geo. Bunyard, Pale Lilac

Rose ...................4. 4.50

6.50

36.00

8.2 Mrs. John M. Lewis, Oxblood Red. 2.50

7.6 Norfolk, Pale Pink............ 1.00

8.5 Octavie Demay, Pink and White. 1.00

8.8 Odette, Rose and Pink........ 1.50

7.6 Old Silver Tip, Madder Red.... 1.00

8.5 Opal, Opalescent Tints........ 2.50

Othello, Deep Crimson......... 4.50

8.7 Paradise, Hydrangea Pink...... 7.50

Paul Fischer, Lilac Rose....... 1.50

8.4 Pasteur, Rose Pink............ 2.00

7.0 Petite Renee, Light Magenta..... 1.25

7.9 Perfection, Pale Shell Pink..... 1.25

8.8 Phoebe Carey, Lavendar Pink... 5.50

9.2 Philippe Rivoire, Dark Red.....12.50

7.7 Philomele, Yellow to Cream.... 1.00

9.0 Phyllis Kelway, Pale Pink...... 7.50

7.6 Pierre Dessert, Crimson Silver-

tipped .................... 1.00

7.6 Pierre Ducharte, Lilac Pink..... 1.50

7.4 Pierre Reignoux, Brilliant Red.. 1.50

8.6 Primevere, Nearest to Yellow.... 2.50

7.4 Princess Beatrice, Violet Rose... 1.00

9.3 Pres. Wilson, Rose Pink........ 35.00

8.9 Pride of Essex, Deep Flesh Pink. 8.00

7.5 Prince of Darkness, Very Dark

Red ..................... 1.00

8.00

6.00

2.50

2.50

3.50

2.50

6.00

8.00

18.00

3.50

4.75

3.00

3.00

13.25

2.50

18.00

2.50

3.50

3.50

6.00

2.25

84.00

19.00

7.7 Pres. Roosevelt, Fine Red...... 1.00

Queen of the Pleasance, Rose and

Sal. .................... 2.00

7.2 Queen Victoria, Cream White... 1.00

2.50

2.50

4.75

1.50

8.3 Rachel, Soft Flesh Pink.........4.00

9.0 Raoul Dessert, Mauve Pink...... 7.50

9.50

18.00

8.7 Reine Hortense, Light Shell Pink. 1.75

8.8 Richard Carvel, Brilliant Red... 4.00 
9.0 Rosa Bonheur, Flesh Pink..... 3.75

7.0 Rose D'Armour, Soft Pink....... 1.00

8.8 Rosette, Salmon Pink..........4.75

7.2 Rubra Superba, Dark Red..... 1.00

7.6 Ruth Brand, Lav. Pink......... 1.25

7.1 Ruy Blas, Pure Mauve......... 1.25

9.0 Sarah Bernhardt, Pink........ 2.00

7.7 Sister Anne, Shell Pink......... 1.00

2.50

7.6 Souv. De L'Expos. Universelle, Violet Rose ............... 1.00

9.1 Souv. De Louis Bigot, Bengal Rose .................. 5.00

9.7 Solange, Lilac White Hav. Brown 4.50 Solfatare, Amber Yellow....... 1.00

7.2 Sou. De Dr. Bretonneau Rose.... 1.00

7.8 Sou. De L'Exposition De Bordeau

Shell Pink and Rose........... 1.00

8.2 Splendida, Pink ........... 2.00

8.5 Standard Bearer, Light Pink.... 7.00

8.0 Stephanie, Del. Pink.......... 1.50

7.9 Simonne Chevalier, Bri. Pink.... 1.00

7.7 Suzanne Dessert, Pure Mauve... 1.50

8.2 Suzette, Bengal Rose......... 1.75

9.7 Therese, Soft Pink........... 3.75

8.0 Tragedie, Dark Crimson........ 2.75

8.2 Triomphe De L'Exposition De

Lille, Shell Pink............. 1.00

4.25

9.00

6.50

9.4 Tourangelle, Shell Pink........ 3.00

7.4 Umbellata Rosea, Rose.......... 1.00

8.5 Venus, Shell Pink............ 1.50

7.0 Virgo Marie, Pure White....... 1.00

8.2 Victoire De La Marne, Red..... 3.00

9.2 Walter Faxon, Bright Rose..... 4.50

7.8 Welcome Guest, Bright Rose.... 1.50 8.4 Wm. F. Turner, Dark Crimson.. 3.25

7.9 Wm. Penn, Light Rose........ 1.00

8.3 Winifred Domne, Crimson...... 2.75

\section{SINGLES}

8.5 Albi Flora, White........... 1.50

Brightness, Peach Pink........ 1.25

Countess of Altemont, Flesh..... 1.25

8.2 Darkness, Maroon ............ 1.25 
8.6 La Jour, White............ 3.50

8.50

Miss Brice ................ 1.25

Miss Ida Chamberlain ....... 1.25

8.9 Pride of Langport, Peach Pink. 5.00

Queen Mary, White.......... 1.25

8.5 Rosy Dawn, Blush White...... 1.50

8.5 Stanley, Bril. Crimson......... 1.25

8.5 The Queen, White ............ 1.50

3.50

8.5 The King, Crimson ........... 1.50

8.0 The Moor, Garnet........... 1.50

8.6 Nellie, Pink ................. 3.00

7.25

8.5 Wild Rose, Pink............ 1.50

3.50

\section{JAP. PEONIES}

Albatross, Pink and Old Gold.... 1.25 3.00

8.2 Attraction, Rose and Yellow... 2.00 4.75

7.7 Apple Blossom, Blush-Yellow.... 1.50

Crystal Queen, Pure White..... 1.50

Dawn, Soft Pink.............. 3.00

7.25

Daybreak, Soft Pink.......... 2.00

8.3 Innocence, Lilac Rose.......... 1.50

9.3 Isami Giden, Pure White......10.00

8.6 King of England, Rich Ruby.... 4.00 10.75

8.9 Marguerite Atwood, Pure White.. 5.00 12.00

8.6 Mikado, Dark Crimson........ 2.00

7.6 Petite Renee, Rose Magenta..... 1.50

Queen Alexandra, Pale Pink.... 1.50

8.5 Rosalind, Pink ............. 1.50

8.9 Tokio, Pink ............... 3.50

Sunrise, Yellow ............ 2.50

9.2 Tuyajo, Vel. Mahogany........10.00

9.4 Tamatbako, Lilac Pink........25.00

9.0 White Lady, White Large..... 5.00

12.00

\section{OFFICINALIS AND RELATED VARIETIES}

3.2 Rosea Superba, Pink........\$1.50

$\$ 3.50$

S.6 Rubea Plena, Brilliant Red..... 1.50

8.5 Tennuifolia Fl. Plena, Red..... 2.00

8.6 Le Printemps ............. 1.50 


\section{RARE PEONIES}

Below is a complete list of the new Brand and Vories Peonies which we are offering to our customers for delivery this fall.

\section{BRANDS}

Blanche King, Immense Deep Pink.....\$50.00 Ella Christensen, Early Deep Pink..... 50.00 Hansina Brand, Deep Pink........ 50.00 Hazel Kinney, Pure Delicate Pink..... 50.00 Laverne Christensen, Pink......... 50.00

9.6 Mrs. A. M. Brand, Immense Pure White 50.00 Mrs. F. A. Goodrich, Deep Pink..... 50.00 Mrs. Frank Beach, Very Large Cream White ...................... 50.00 Mrs. Harriet Gentry, Pure White..... 50.00 Mrs. John M. Kleitsch........... 50.00 Mrs. Romain B. Ware, Flesh Pink..... 50.00 Myrtle Gentry, Sal. Pink.......... 50.00

9.3 Victory Chateau Thierry, Rose Pink... 50.00

\section{VORIES}

Alex D. Vories, A New Red........ \$15.00 Bishop Burke, Crimson Silver Sheen.... 50.00 Deedie May .................. 5.00

Fanny Lee, American Beauty Red..... 10.00

9.3 Frankie Curtis, White............ 50.00 Henry M. Vories, Light Pink........ 25.00 Lady Kate, Light Pink........... 50.00 Largo ...................... 5.00 Laura Vories ................. 25.00 Mary B. Vories.................. 15.00 9.3 Nancy Dolman, Rose Pink.......... 50.00 


\section{PRIZE WINNING PEONIES}

Below is a list of fine varieties with their ratings in points out of a possible 100 for perfect.

88 Avalanche

86 Marguerite Gerard

91 Martha Bullock

98 Festiva Maxima

89 Claire Dubois

87 Dr. Barnsby

92 Philip Rivoire

87 Jas. Kelway

97 Solange

94 Kelway's Glorious

92 Walter Faxon

99 Le Cygne

88 Alsace-Lorraine

91 Marie Crousse

90 Elwood Pleas

89 Modeste Guerin

83 Mons Dupont

90 Mons Martin Cuhuzac
89 Jeannot

90 Sarah Bernhardt

89 Karl Rosenfield

94 Tourangelle

90 La France

86 Judge Berry

88 Longfellow

95 E. B. Browning

90 Milton Hill

92 Brands Magnificent

92 Mons. Jules Elie

91 Francis Willard

90 Raoul Dessert

92 Jubilee

97 Therese

93 Lady Alex. Duff

87 Reine Hortense

85 Harry Avery

The prize winners in the 1928 Peony Show will be found in this list.

\section{承蛹}

\section{WAHO0 LODGE GARDENS}

BOX 915

SIOUX FALLS, S. DAK. 


\section{MARVELOUS NEW IRIS}

\section{EVENING GLOW}

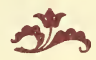

Standards and falls pale silvery blue. Hafts reticulated with brown and very heavy. Orange beards. The lower part of hafts are irridescent in lavender, pink and orange, giving it a wonderful opalescent coloring inside. The foliage is remarkable, being graygreen, the effect being that of a sheen. Stands $24^{\prime \prime}$ to $28^{\prime \prime}$ and full $I^{1} / 4^{\prime \prime}$ to $I \frac{1}{2}$ " blades, beautiful with no flowers. The flowers are large, but not extraordinary, larger often than Brandywine which at first glance it somewhat resembles. In all a robust vigorous fine Iris, $32^{\prime \prime}$ to $36^{\prime \prime}$, three to six flowers open at once.

For distribution in 1927 at \$10.00. A limited number of orders booked now for 1927 delivery. Cash must accompany order.

Introduced by

\section{Wahoo Lodge Gardens Sioux Falls, S. D.}


